\title{
On-line identification, flutter testing and adaptive notching of structural mode parameters for V-22 tiltrotor aircraft
}

\author{
R K MEHRA ${ }^{1}$, P O ARAMBEL ${ }^{1}$, A M SAMPATH ${ }^{1}$, \\ R K PRASANTH ${ }^{1}$ and T C PARHAM ${ }^{2}$ \\ ${ }^{1}$ Scientific Systems Company, Inc. (SSCI), 500 West Cummings Park, Suite \\ 3000, Woburn, MA, 01801, USA \\ ${ }^{2}$ Bell Helicopter Textron, Inc. (BHTI), PO Box 482, Fort Worth, TX 76101, \\ USA \\ e-mail: \{rkm, pablo, arun, prasanth\}@ssci.com; \\ TParham@BellHelicopter.textron.com
}

\begin{abstract}
New algorithms and results are presented for flutter testing and adaptive notching of structural modes in V-22 tiltrotor aircraft based on simulated and flight-test data from Bell Helicopter Textron, Inc. (BHTI). For flutter testing and the identification of structural mode frequencies, dampings and mode shapes, time domain state space techniques based on Deterministic Stochastic Realization Algorithms (DSRA) are used to accurately identify multiple modes simultaneously from sine sweep and other multifrequency data, resulting in great savings over the conventional Prony method. Two different techniques for adaptive notching are explored in order to design an Integrated Flight Structural Control (IFSC) system. The first technique is based on on-line identification of structural mode parameters using DSRA algorithm and tuning of a notch filter. The second technique is based on decoupling rigid-body and structural modes of the aircraft by means of a Kalman filter and using rigidbody estimates in the feedback control loop. The difference between the two approaches is that on-line identification and adaptive notching in the first approach are entirely based on the knowledge of structural modes, whereas the Kalman filter design in the second approach is based on the rigid-body dynamic model only. In the first IFSC design, on-line identification is necessary for flight envelope expansion and to adjust the notch filter frequencies and suppress aeroservoelastic instabilities due to changing flight conditions such as gross weight, sling loads, and air speed. It is shown that by tuning the notch filter frequency to the identified frequency, the phase lag is reduced and the corresponding structural mode is effectively suppressed and stability is maintained. In the second IFSC design using Kalman filter design, the structural modes are again effectively suppressed. Furthermore, the rigid-body estimates are found to be fairly insensitive to both natural frequency and damping factor variations and therefore stability is maintained. The Kalman filter design might be a better choice when the rigid-body dynamics are well known because no adaptation is necessary in this case.
\end{abstract}


Keywords. On-line identification; flutter testing; adaptive notching; structural mode parameters; V-22 tiltrotor aircraft.

\section{Introduction}

Design of controllers for complex systems having both rigid body and structural dynamics is usually carried out in two steps. Initially, a feedback controller is designed based only on the rigid body model. Structural modes are then taken care of by inserting appropriate notch filters in the feedback path to avoid those structural modes that destabilize the system through the feedback signals. In some cases, notch filters are placed in the forward path as well to avoid the condition where a spurious feedback through the system's actuators may affect the overall performance. Figure 1 shows the approximate locations of data sensors in a V-22 aircraft during flight testing as well as the different structural modes.

In a V-22 aircraft, the vibrations in the pilot's body can be transmitted through the arm into the stick which can ultimately lead to Pilot Airframe Coupling (Parham et al 1991). Each notch filter's centre frequency is tuned to the natural frequency of the corresponding structural mode. However, the structural mode frequency changes with flight conditions such as gross weight, sling loads, and air speed. Therefore, a notch filter that is designed for certain flight conditions may become ineffective for other conditions. In order to account for these variations, notch filters must be designed wider than required so that they remain effective within the entire range of possible structural mode frequencies. However, the wider the filter band, the larger the phase lag and the worse the performance. The trade-off, therefore, is phase lag vs. robustness to structural mode variations.

This paper describes two design techniques to reduce the phase lag introduced by notch filters while retaining robustness against variations in the natural frequencies and the damping factor of structural modes. Both techniques keep the main rigid body controller unchanged. The first technique is to estimate the structural mode frequency in real time and to adjust the notch filters accordingly. As long as the estimate is accurate enough, the notch filter can be designed for that particular frequency with the narrowest possible band and with the least increase in the overall phase lag. In the second technique, a Kalman filter is used to estimate the rigid body portion of the output; that is, to eliminate the structural components. This estimate is then fed back to the flight control system.

Both schemes are shown in figure 2. The currently used configuration is shown on top. The dotted line represents spurious feedback that affects the pilot's arm. This perturbation can only be suppressed using a notch filter in the forward path. Other notch filters are preferred right after the sensors in the main feedback path. These filters are meant to suppress all the structural vibrations that may affect the stability of the closed loop system. In the centre of the figure is shown the adaptive notch filter design. The configuration is similar to the one currently used, but the identification block provides on-line estimates of structural frequencies which are used to tune the notch filters. In this way, variations on the structural modes are accounted for and narrow filters with small phase lag can be used. At the bottom of the figure, the Kalman filter configuration is shown. The Kalman filter estimates the rigid body portion of the output, which is used to implement the main feedback loop. The notch filter in the forward path cannot be eliminated because that would require the knowledge of a "rigid body model" of the pilot, which is not easy to obtain.

This paper presents a new time-domain approach for structural mode identification (offline and on-line) and a self-adaptive notch filter for the V-22 tiltrotor aircraft. To simplify 


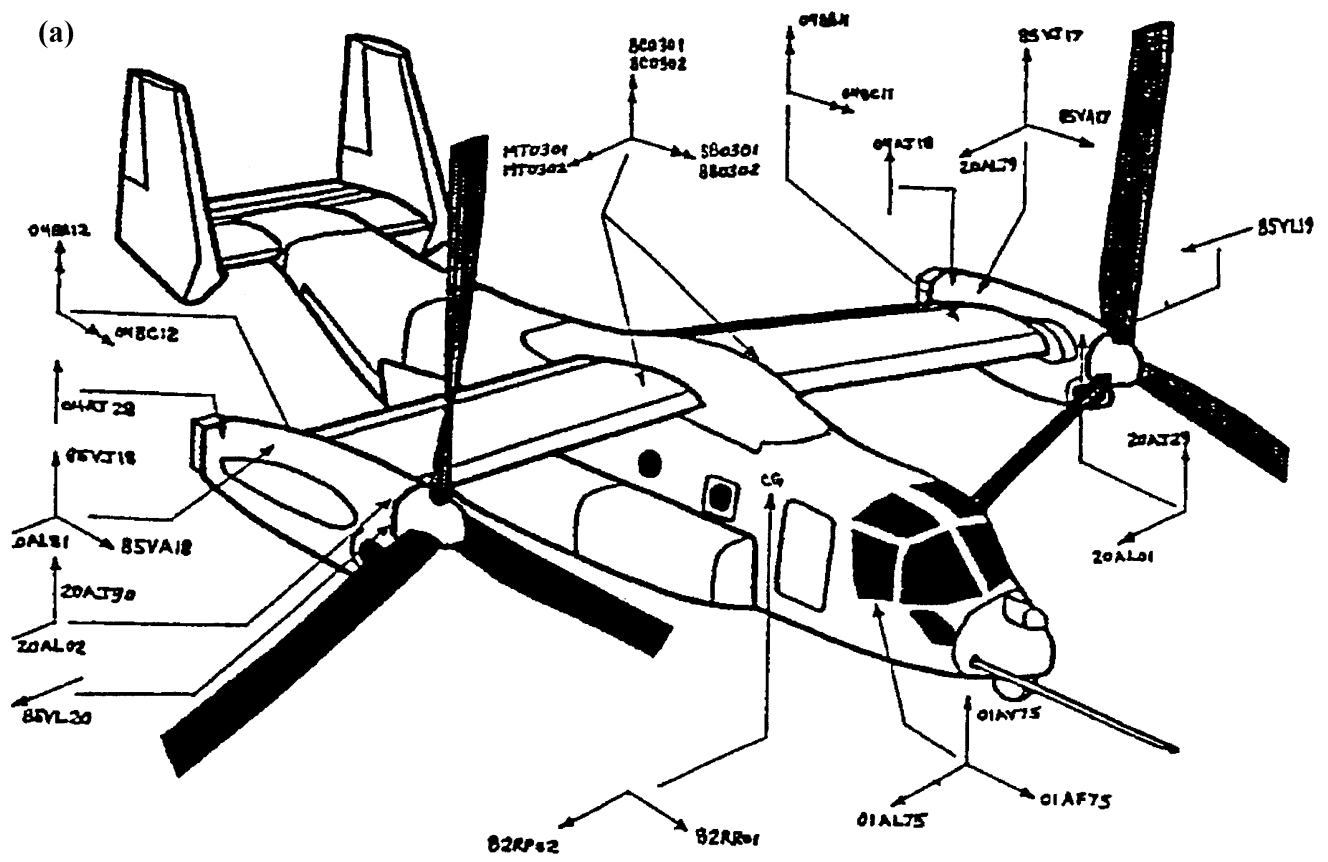

(b)
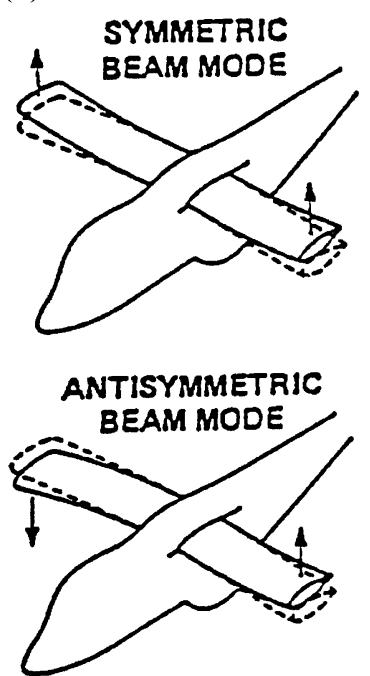
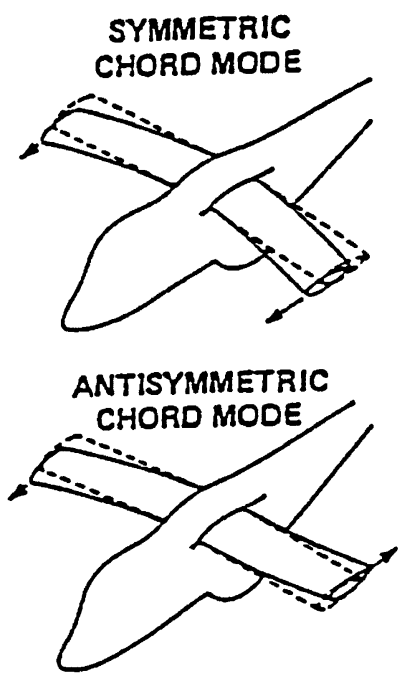

SYMMETAIC TORSION MODE
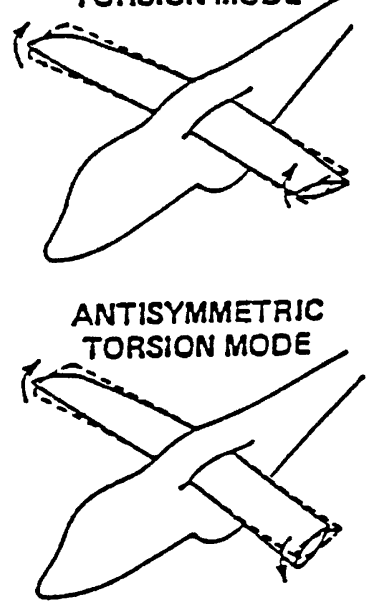

Figure 1. Approximate locations and orientations of data sensors in a V-22 aircraft during flight testing (a) and structural modes in a V-22 aircraft (b).

the discussion, the identification for the flight data is focused on the Symmetric Wing Beam (SWB) mode and for the simulated data is focused on the Antisymmetric Wing Chord (AWC) mode, but similar results can be obtained for other structural modes. The natural frequency of the AWC mode is estimated on-line using a subspace-based deterministic stochastic realization algorithm (DSRA) (Mehra et al 1998). This estimate is used to update 
(a)

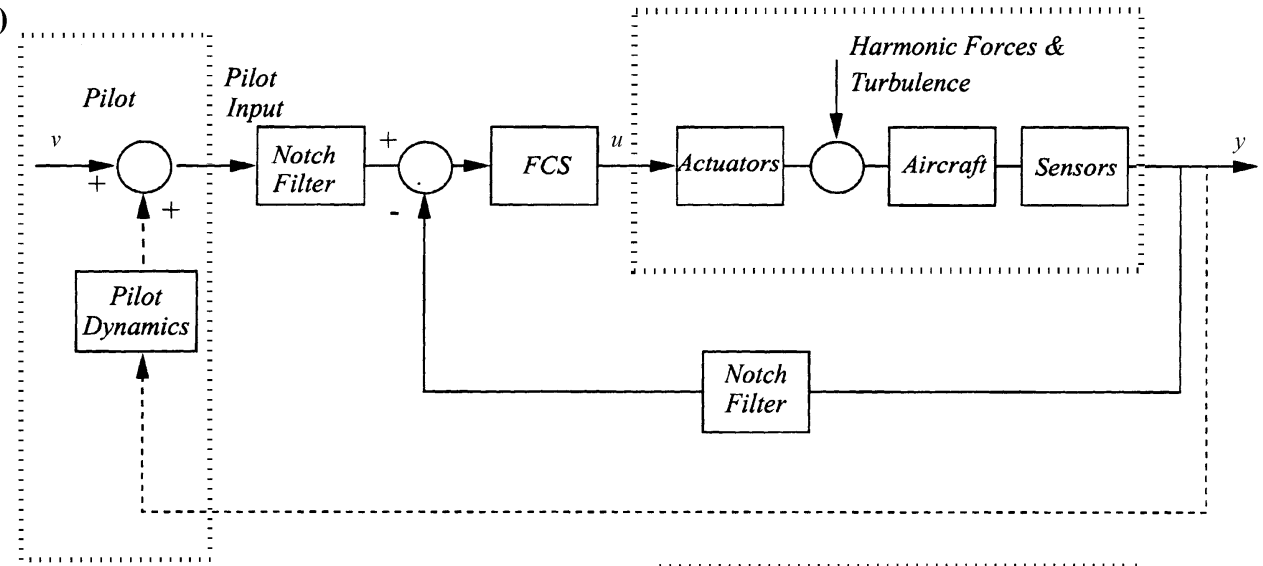

(b)

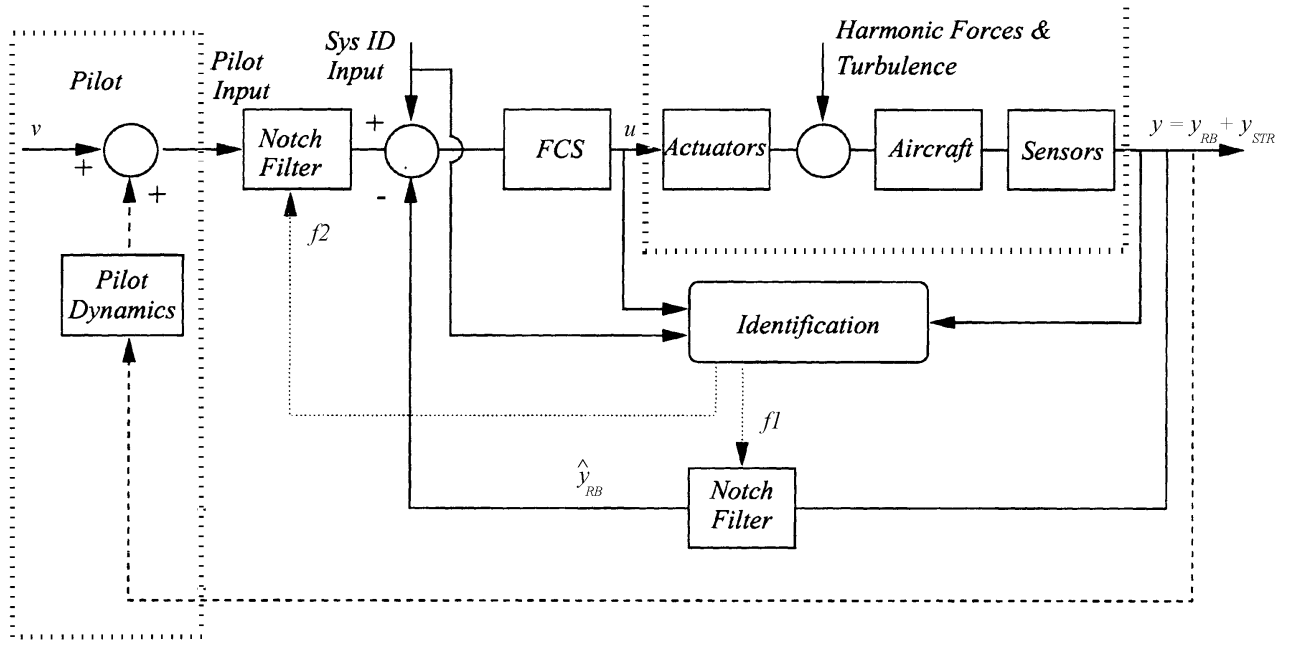

(c)

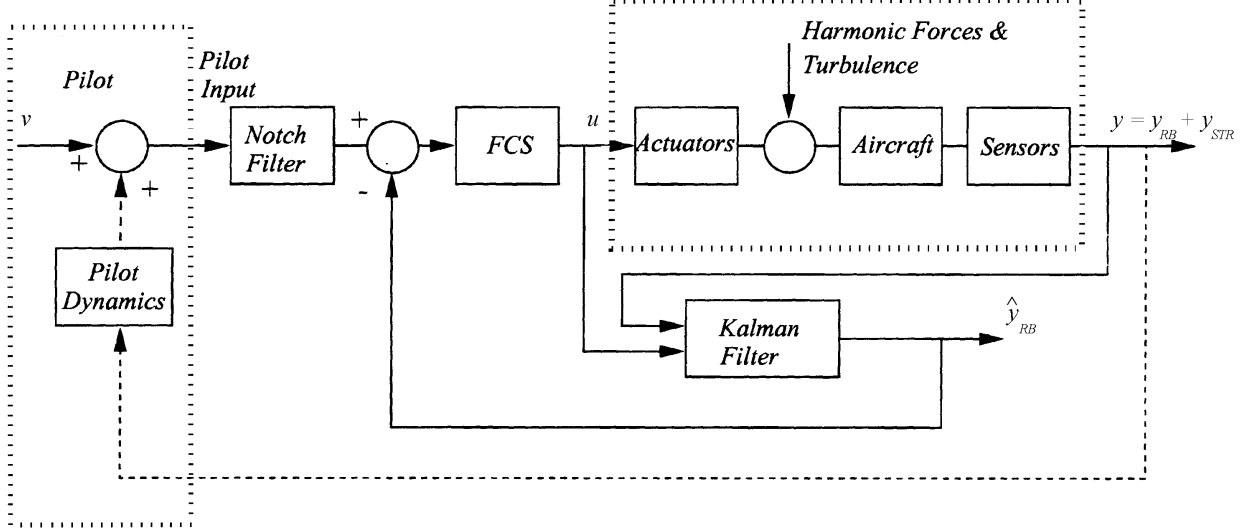

Figure 2. Current (a), notch filter (b) and Kalman filter (c) configurations for structural mode suppression. 
the notch filter's centre frequency. During flight testing, DSRA is used to simultaneously estimate multiple structural modes depending on the input signal, and this information can be used in near real-time for on-line flight envelope expansion.

This paper is organized as follows. In the next section, the details of the DSRA algorithm are presented. In $\S 3$, flight test results are presented. Adaptive notch filter design is discussed in $\S 4$. In $\S 5$, simulation results are presented for the entire system including the notch filter adaptation. The Kalman filter configuration is described in $\S 6$ and, finally, a few conclusions are stated in $\S 7$.

\section{DSRA identification algorithm}

The evolution of SRA and DSRA algorithms as well as those of selected N4SID and MOE algorithms is discussed in the literature (Akaike 1974; Aoki 1987; Faure 1976; Mehra 1991, 1994; van Overschee \& De Moor 1993, 1994; Viberg et al 1993; Verhaegen 1994). Identification is carried out with a deterministic-stochastic realization algorithm (DSRA), based on the subspaces of certain Hankel matrices constructed from input and output data.

Identification problem: Let $N$ be a positive integer and $\left\{u_{k}, y_{k}\right\}_{k=1}^{N}$ be a collection of multi-input-multi-output data generated by a state space causal stable discrete-time LTI system $G$ of the form:

$$
\begin{aligned}
x(k+1) & =A x(k)+B u(k)+w(k) \\
y(k) & =C x(k)+D u(k)+v(k)
\end{aligned}
$$

where $x \in R^{n}$ is the state vector, $u \in R^{n u}$ is the control input vector, $y \in R^{n y}$ is the output vector, $w \in R^{n}$ and $v \in R^{\text {ny }}$ are white noise input vectors of strength

$$
E\left(\left[\begin{array}{c}
w \\
v
\end{array}\right]\left[\begin{array}{ll}
w^{\prime} & v^{\prime}
\end{array}\right]\right)=\left[\begin{array}{ll}
W & S \\
S^{\prime} & V
\end{array}\right] \geq 0 .
$$

The open loop system identification problem is to compute a state space model for the system $G$, that is, find matrices $A, B, C, D, W, S, V$ and state vector dimension $n$ such that the observed data is generated by the system in (1) and (2) with noise covariances given by (3).

DSRA algorithm: Equations (1) and (2) can be written in terms of past and future vectors as follows:

$$
Y_{f}=L_{p}\left[\begin{array}{c}
U_{p} \\
Y_{p}
\end{array}\right]+T_{d} U_{f}+\text { noise }
$$

and the system matrices $A, B, C$ and $D$ can be derived from the matrices $L_{p}$ and $T_{d}$. One of the versions of the DSRA algorithm is shown in figure 3 and involves the following steps.

(1) The following minimization problem for $L_{p}$ and $T_{d}$ is solved:

$$
\min _{L_{p}, T_{d}}\left\|Y_{f}-L_{p}\left[\begin{array}{c}
U_{p} \\
Y_{p}
\end{array}\right]-T_{d} U_{f}\right\|_{F}
$$




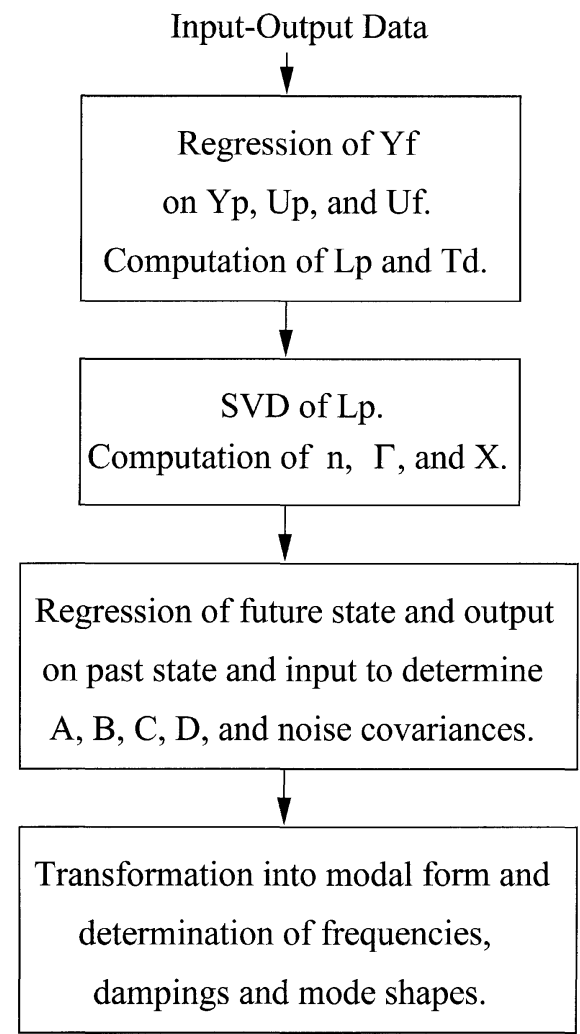

Figure 3. Major steps in DSRA.

where $Y_{p}, U_{p}, Y_{f}$, and $U_{f}$ are Hankel matrices built of the past output, the past input, the future output, and the future input respectively. For example, $Y_{p}$ is given by:

$$
Y_{p}=\left[\begin{array}{cccc}
y_{1} & y_{2} & \cdots & y_{N-2 n i} \\
y_{2} & y_{3} & \cdots & y_{N-2 n i+1} \\
\cdot & \cdot & \cdots & \cdot \\
\cdot & \cdot & \cdots & \cdot \\
y_{n i} & y_{n i+1} & \cdots & y_{N-n i}
\end{array}\right],
$$

where $N$ is the record length (that may grow with time) and $n i$ is a design parameter representing both the past and the future frame lengths.

(2) The (ordered) SVD of $L_{p}$ is performed, and the order $n$, the extended observability matrix $\Gamma$, and the Kalman state vector estimates $X=\left[x_{n i+1} \cdots x_{N-n i+1}\right]$ are computed.

(3) The system matrices $A, B, C$, and $D$ are obtained by solving the following least squares problem:

$$
\min _{A, B, C, D}\left\|\left[\begin{array}{lll}
x_{n i+2} & \cdots & x_{N-n i+1} \\
y_{n i+1} & \cdots & y_{N-n i+1}
\end{array}\right]-\left[\begin{array}{cc}
A & B \\
C & D
\end{array}\right]\left[\begin{array}{lll}
x_{n i+1} & \cdots & x_{N-n i} \\
u_{n i+1} & \cdots & y_{N-n i}
\end{array}\right]\right\| .
$$

(4) The system is transformed into modal form using eigenvalues and eigenvectors of $A$. Estimates of frequencies, dampings and mode shapes are obtained from these eigenvalues and eigenvectors. 
Other forms of the DSRA algorithm developed at SSCI include: the SRA algorithm, which is applied when there is no forced excitation; DSRA-covariance, which bases the computations on estimated data covariances; DSRA batch, which avoids the use of the SVD algorithm; DSRA QR, which is based on the QR decomposition algorithm; and DSRA-on-line, which performs the computations recursively and is suitable for real-time identification. DSRA estimates can be further refined using maximum likelihood estimation (MLE) and Cramer-Rao bounds on parameter covariances can be obtained (see Mehra 1994). It should also be mentioned that DSRA can also identify rigid-body modes with proper excitation inputs.

\section{DSRA on flight test data}

For the V-22 aircraft, the primary method of verifying the damping in the airframe structural modes used by Bell Helicopters was to excite each individual mode with a sine dwell and calculate the damping from the decay using the Prony curve fit method. This is not only time-consuming but is also very expensive and cumbersome. In contrast, the chief

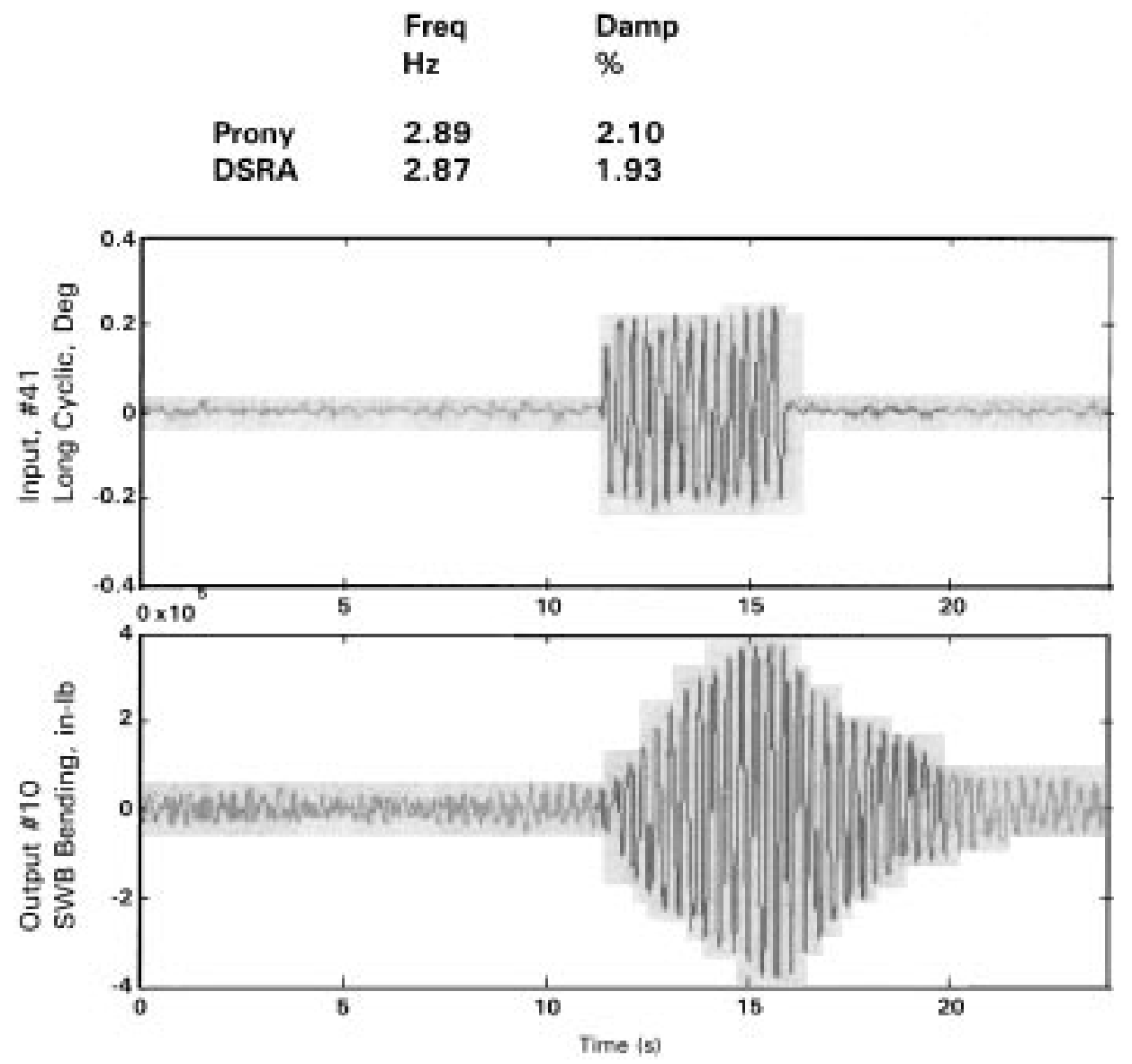

Figure 4. Typical SWB excitation, symmetric wing beam bending output. 
DSRA Batch Results

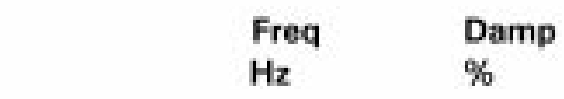

$\begin{array}{lll}\text { SWB } & 2.83 & 2.86 \\ \text { SWT } & 5.18 & 4.58\end{array}$
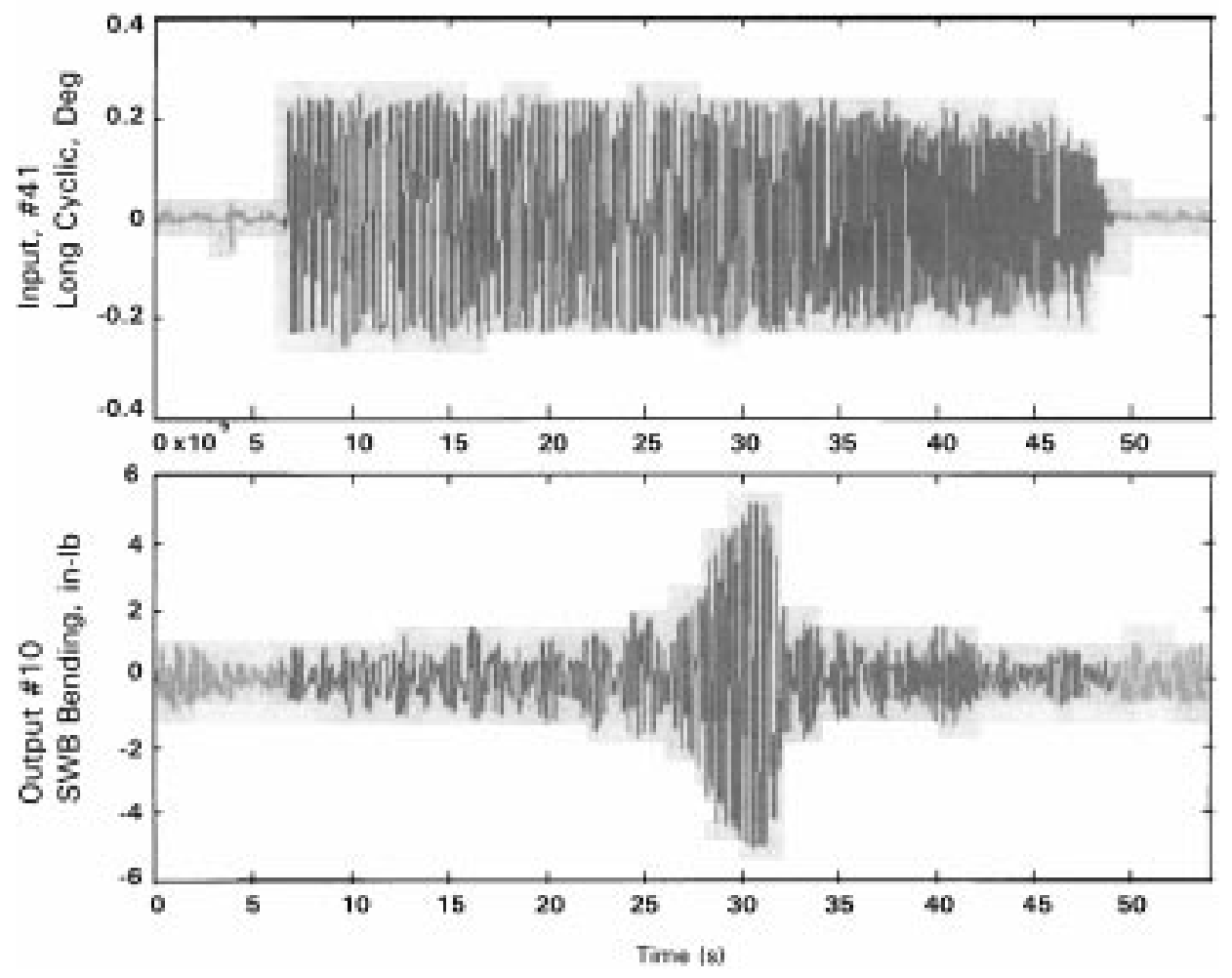

Figure 5. Longitudinal cyclic sine sweep.

advantage of using the DSRA method is that it can identify multiple modes from a single sine sweep excitation. The DSRA was evaluated on V-22 EMD flight test data for the critical antisymmetric wing chord (AWC) mode as well as the symmetric wing beam (SWB), symmetric wing chord (SWC), and symmetric wing torsion (SWT) modes and the estimated frequency and damping values were compared with those obtained using the Prony method. The results show some scatter in the measured data but overall, the estimates from the Prony method and the DSRA method agree very well. The DSRA method was also evaluated on the limited sine sweep data that was available. These data show that the DSRA estimates for multiple modes during a single sine sweep agree very well with the estimates from sine dwells. This represents great savings on flight test time, particularly during high speed dive maneuvers which are very demanding on the pilot. These results are presented in figures 4-8 and are discussed next.

Comparison of DSRA and Prony approaches: Figure 4 shows a typical SWB sine dwell excitation and decay for Test 353 Record 46 at 150 knots airspeed. The input for this case is 


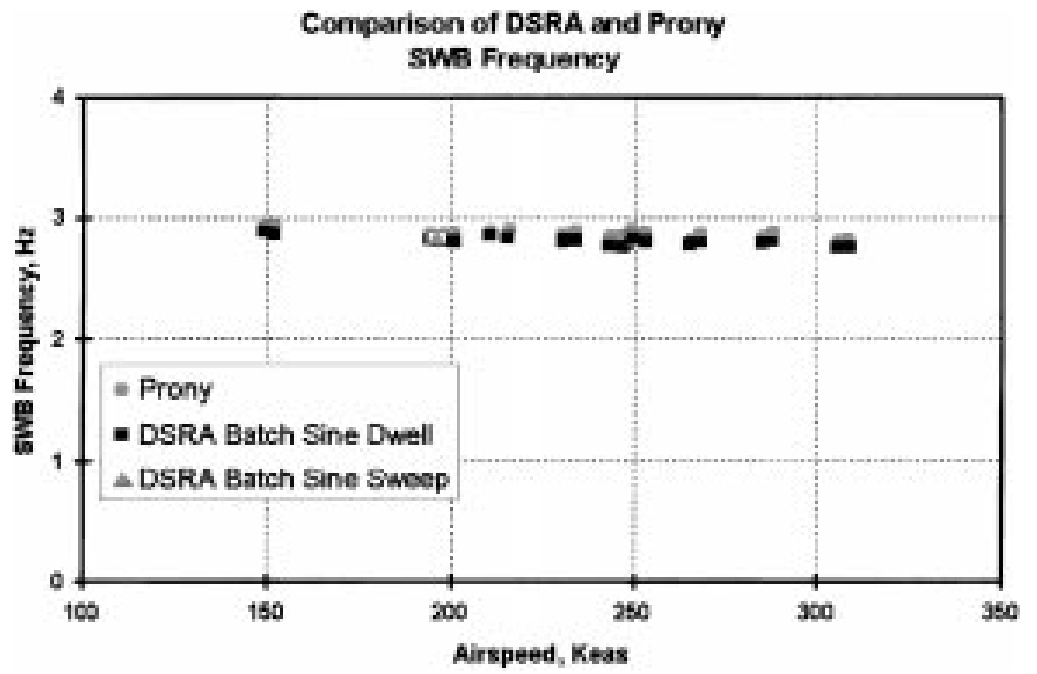

Figure 6. Comparison of DSRA and Prony frequency estimates for SWB mode.

longitudinal cyclic and the output is symmetric wing beamwise bending. The Prony estimate for this case is $2.89 \mathrm{~Hz}$ and $2.10 \%$ damping. The DSRA estimates for this condition are $2.87 \mathrm{~Hz}$ and $1.93 \%$ damping. Thus the two methods agree very well.

Figure 5 shows a longitudinal cyclic sine sweep excitation from $2 \mathrm{~Hz}$ to $8 \mathrm{~Hz}$. From this single excitation, DSRA estimated the SWB mode frequency at $2.83 \mathrm{~Hz}$ and damping at $2.86 \%$ with corresponding values for the SWT mode at $5.18 \mathrm{~Hz}$ and $4.58 \%$. For this record, there is no Prony estimate to compare with since the Prony method works only with unforced decays. Figure 6 compares the Prony and DSRA estimates for the SWB mode frequency for the available test data. On the plot are the Prony (circle) and DSRA (square) estimates for many sine dwell points. For the sine dwell data, DSRA and Prony agree very

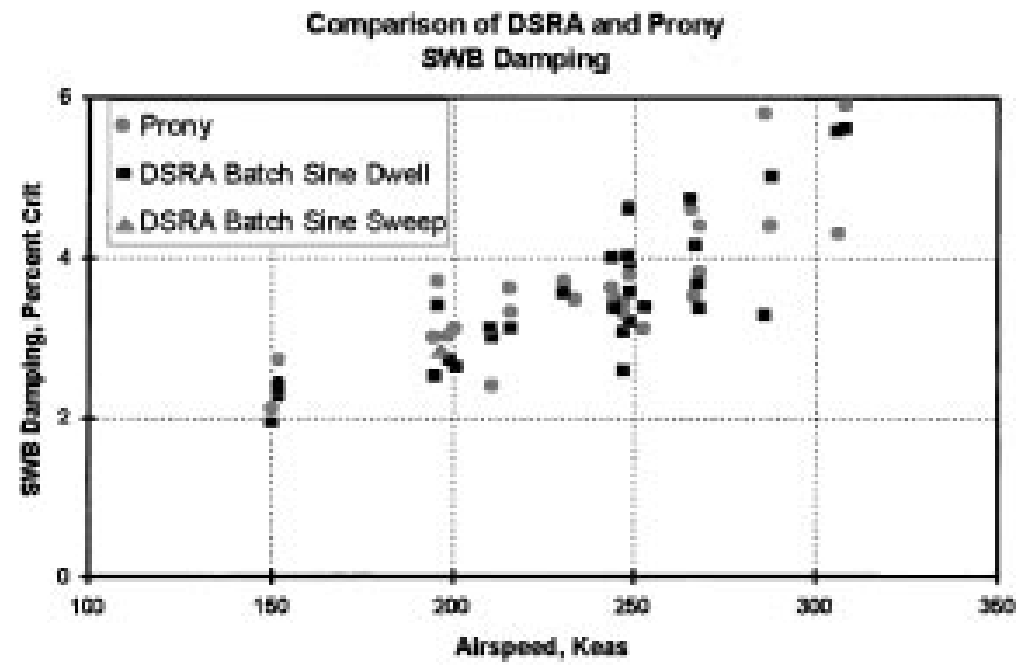

Figure 7. Comparison of DSRA and Prony damping estimates for SWB mode. 


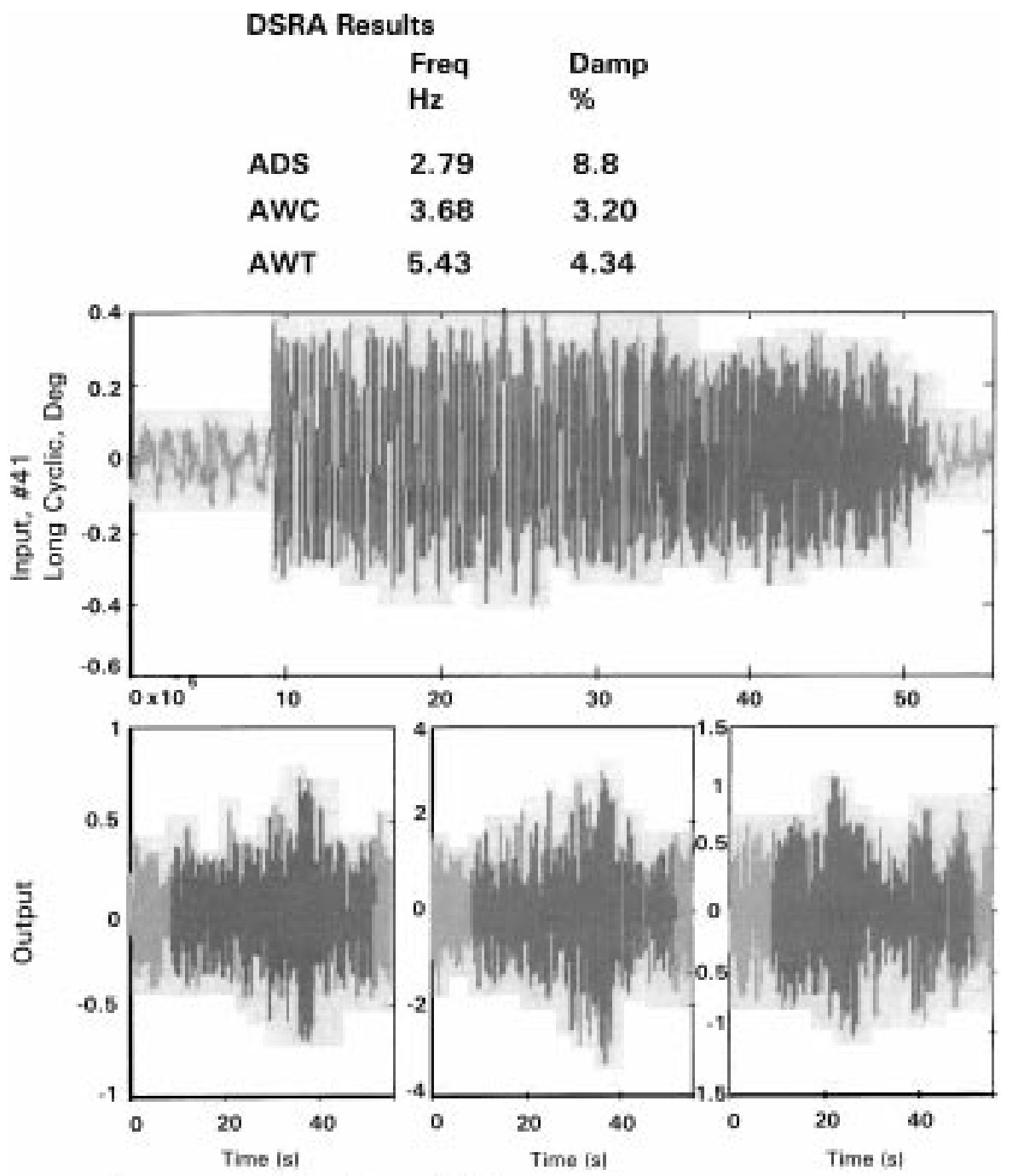

Outputs are \#7, \#9, and \#21 AWC Bend (ib-lb), AWT Bend (in-lb), and Mast $\mathrm{Q}$, (in-lb)

Figure 8. Helicopter mode sine sweep with three outputs.

well. There is only one sine sweep available (figure 5) which is plotted as a triangle in figure 6. Again, this DSRA estimate from the sine sweep agrees very well with the sine dwell data. Figure 7 shows the same DSRA vs. the Prony correlation for the corresponding damping data. Although there is more scatter in the damping estimates, the DSRA and Prony results agree very well. The single sine sweep point falls within the data scatter at 200 knots. Although there are limited SWT data to compare with the SWT sine sweep estimates in figure 5, this estimate does agree with the data available.

An additional advantage of DSRA over Prony is the ability of DSRA to handle more than one input. Figure 8 shows a helicopter mode sine sweep excitation that was analysed with 
three outputs. The DSRA analysis finds three modes of the airframe. The results agree with the sine dwell estimates from Prony.

Based on these comparisons of the DSRA method with Prony, the following conclusions can be drawn: (1) DSRA estimated frequency and damping from sine dwells are in good agreement with Prony results; (2) DSRA estimated frequency and damping from sine sweeps agree well with Prony and DSRA sine dwell data; (3) With DSRA, sine sweeps and other inputs can be used to estimate more than one mode simultaneously, and (4) DSRA and Prony are both sensitive to signal-to-noise ratio (SNR), which relates to fundamental issues of identifiability. The design of proper input signals to improve SNR is an important topic and is surveyed by Mehra (1994). Next, results obtained using on-line identification and adaptive notching on simulated flight test data are presented in $\S \S 4$ and 5 .

\section{Adaptive notch filter}

The AWC mode of the V-22 aircraft with a resonance frequency of about $3.3 \mathrm{~Hz}$ is a critical mode responsible for Pilot Airframe Coupling (PAC) and is the focus of this study.

How the AWC mode can cause instability: The excitation of the AWC mode causes lateral acceleration at the location of the pilot seat, which when fed back into the system through the pilot's arm and the lateral movement of the stick results in undesired commands to the ailerons. The aileron deflection, in turn, causes a sidewash velocity at the vertical fins resulting in a side force on the fins, which further reinforces the oscillations at the AWC mode frequency. This repetitive process results in a "resonance effect" and can destabilize the aircraft.

Notch filter configuration: In figure 2, the feedback path that is relevant for the AWC mode related instability analysis is the one that includes the pilot dynamics block and the forwardpath notch filter. Within the frequency range corresponding to the AWC mode, the rigid body dynamics has little effect on structural mode dynamics; hence, the analysis and simulations are carried out using the simplified diagram of figure 9, which only includes the pilot seat lateral acceleration output, the pilot dynamics block, and the forward-path notch filter. The block $\mathrm{G}(\mathrm{s})$ in figure 9 represents the structural dynamics. The feedback block $\mathrm{H}(\mathrm{s})$ represents the flight control system, the actuators and the sensors, as well as the pilot dynamics.

Notch filter requirements: The general requirements that the forward-path notch filter must satisfy are as below.

Stability - The filter should be designed so that the overall feedback system described in figure 9 (and in figure 2) is stable.

Good disturbance rejection in the AWC frequency region $(3 \mathrm{~Hz})$ - To minimize the effect of the AWC mode on pilot airframe coupling, the closed loop transfer function from the lateral force on the vertical fin to the pilot seat lateral acceleration should be minimized. This, in turn, means that the magnitude of this transfer function should be as small as possible around $3 \mathrm{~Hz}$ (the frequency of the AWC mode).

No distortion of pilot commands below $1.5 \mathrm{~Hz}$ - The commands that the pilot wishes to give to the ailerons should not be distorted by the filter $\mathrm{F}(\mathrm{s})$. Taking into consideration how fast 


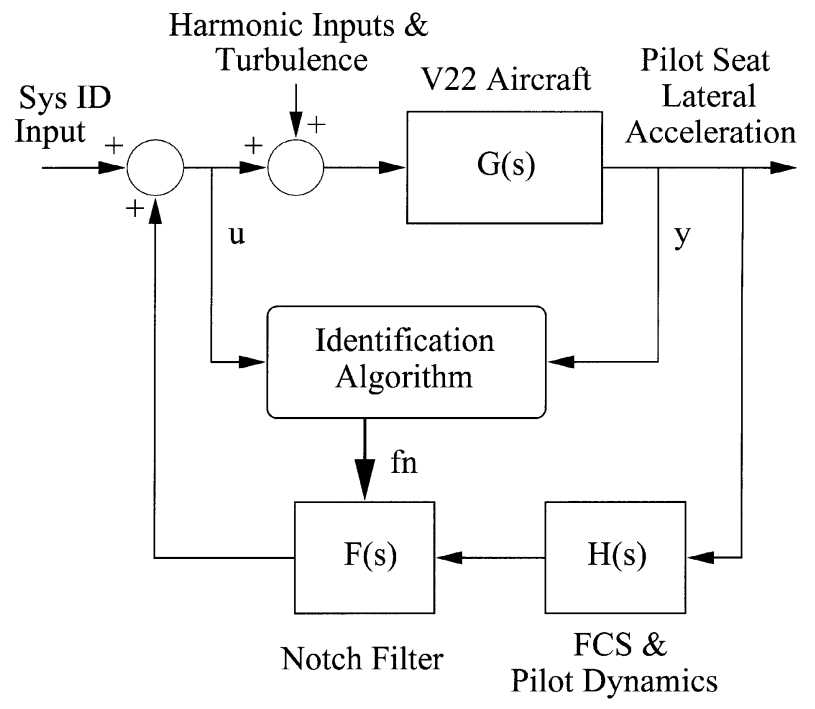

Figure 9. Simplified diagram for the study of the AWC mode.

the pilot may be able to think and react, this desired property can be restated that the magnitude of the filter $\mathrm{F}(\mathrm{s})$ should be very close to unity within the frequency range from 0 to $1.5 \mathrm{~Hz}$.

Good speed of response and minimal delay - The filter should not introduce any delay and respond as quickly as possible to the pilot's command. This criterion can be captured by the speed of response to a step. This criterion also implies that there be minimal phase lag at low frequencies.

Notch filter family: A family of notch filters that satisfies the previous requirements is given by:

$$
F(s)=\frac{s^{2}+s+4 \pi^{2} f_{n}^{2}}{s^{2}+4 s+4 \pi^{2} f_{n}^{2}},
$$

where $f_{n} \mathrm{~Hz}$ is the natural frequency of the filter, which is tuned to the estimated AWC mode. For this application, it is sufficient to update only $f_{n}$, while the other terms in the denominator and the numerator are constant. The bandwidth of these filters is narrow enough to have little impact on the overall phase lag. However, a change of $\pm 0.2 \mathrm{~Hz}$ in the AWC natural frequency creates instability, unless the centre frequency, $f_{n}$, is adjusted accordingly. The identification algorithm, therefore, must track the changes in the AWC mode frequency and provide the $f_{n}$ for notch filter adaptation.

\section{Simulations}

The Sys ID signal in figure 9 (or figure 2) is a set of sinusoids in the range of 2.5 to $4 \mathrm{~Hz}$. This signal is necessary to perform the identification because the system is operating in a closed loop. The estimated frequency $f_{n}$ of the AWC mode is used to tune the notch filter 

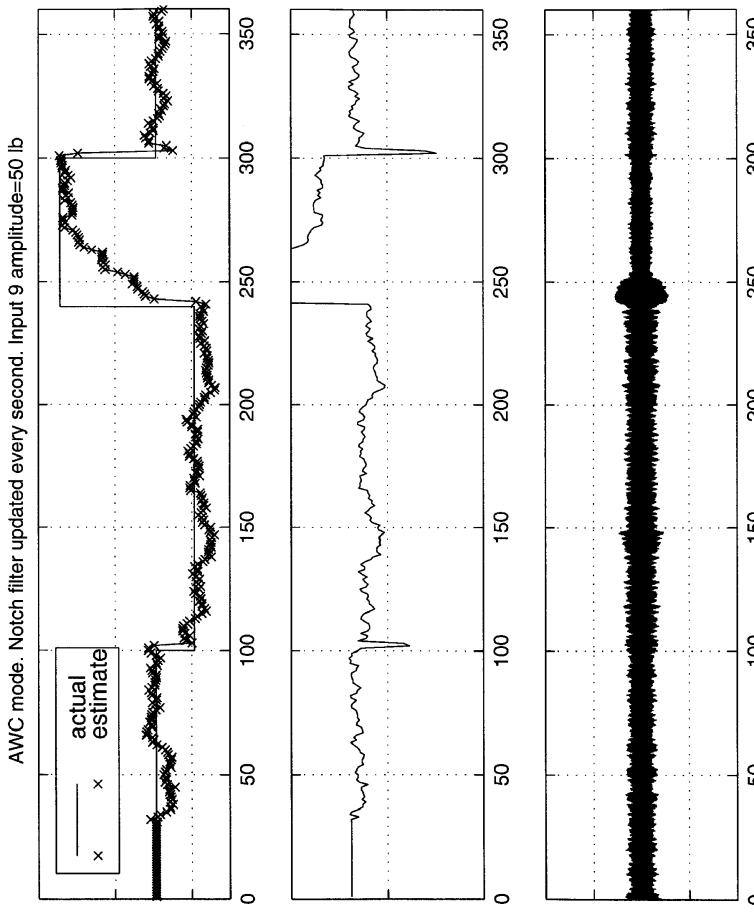

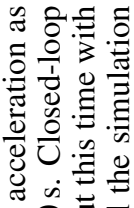

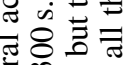

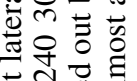

조유

क응

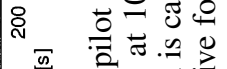



-

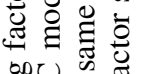

政蛋

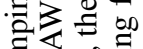

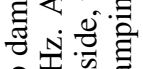

을 च

ㅇํํ코

ठृ

응 $\approx$.
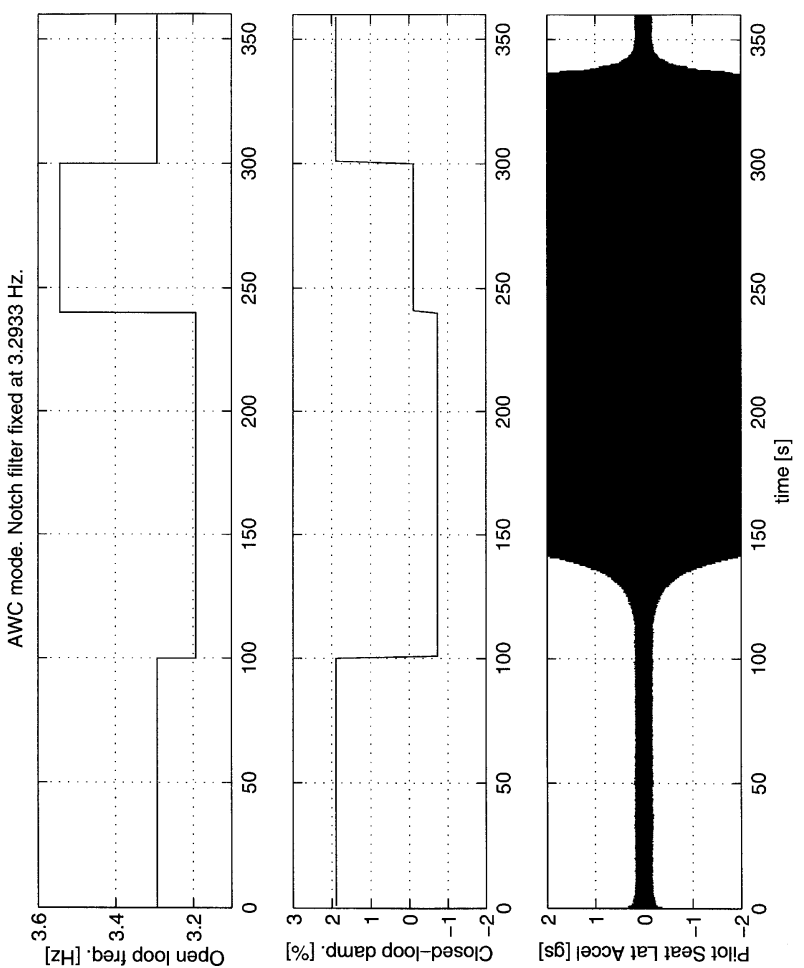

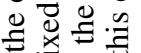

元 0

\&

는웡

능응

过

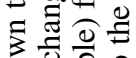

요 0

电氖

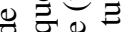

施

응

ت응

뒁ㅎㅇ

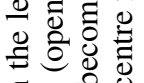

ธิ

导売

$\dot{\theta}$

(1)

这交言

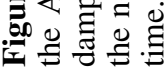


every second; the sampling time is close to $0.01 \mathrm{~s}$. A forgetting factor of 0.9990 is used. Two kinds of simulations are carried out. In the first, it is assumed that the AWC mode frequency changes abruptly at certain instants of time; in the second simulation, the variation in frequency change is assumed to be smooth and linear. Here, results are presented only for the first case, for brevity. The changes in the AWC mode frequency are assumed to follow the pattern in figure 10. The notch filter's centre frequency is fixed at $3.2933 \mathrm{~Hz}$. As the actual AWC mode frequency changes, the closed-loop damping changes, going from almost $2 \%$, down to $-0.8 \%$, to $-0.1 \%$ and back again to almost $2 \%$. Negative damping factors correspond to unstable systems; therefore, the output signal starts to grow until the system becomes stable again. Both the closed-loop damping and the output signal are also shown in figure 10. In the next simulation, the notch filter is updated according to the estimated frequencies. The probe signal amplitude is fixed at $50 \mathrm{lb}$. The results are shown in the right hand side of figure 10. The AWC mode frequency estimate is sufficiently accurate such that the closed-loop damping is positive most of the time. Only during the transitions, the notch filter is not tuned to the right frequencies, and the output amplitude increases. One could use a larger probe signal amplitude to speed up the convergence to the right frequency but the trade-off is that the output due to the probe signal may become undesirably large.

\section{Kalman filter configuration}

The second design considered in this paper uses a Kalman filter to filter out the structural modes. This structure is more robust to uncertainties in the structural modes because it relies entirely on the rigid body portion of the model. In addition, since it does not require adaptation as opposed to the previous approach, the possibility of divergence of the algorithms is greatly reduced. The simulations were carried out using a Simulink model of the rotorcraft with two inputs - rudder and aileron - and the three outputs that are fed back into the flight control system, namely, lateral acceleration sensor, roll rate sensor, and yaw rate sensor. As shown in figure 2, the entire system also includes the flight control system, whose inputs are the following pilot commands: the lateral stick and the pedal.

\subsection{Kalman filter design}

The full analytical model has 6 states that correspond to rigid body dynamics and 42 states that correspond to structural modes. The design is based upon the rigid body portion of order 6 . The first step is to bring the entire model to a modal form and group those states that correspond to the rigid body:

$$
\begin{aligned}
{\left[\begin{array}{c}
\dot{x}_{\mathrm{RB}} \\
\dot{x}_{\mathrm{Str}}
\end{array}\right] } & =\left[\begin{array}{cc}
A_{\mathrm{RB}} & 0 \\
0 & A_{\mathrm{Str}}
\end{array}\right]\left[\begin{array}{c}
x_{\mathrm{RB}} \\
x_{\mathrm{Str}}
\end{array}\right]+\left[\begin{array}{c}
B_{\mathrm{RB}} \\
B_{\mathrm{Str}}
\end{array}\right] u, \\
y & =\left[\begin{array}{ll}
C_{\mathrm{RB}} & C_{\mathrm{Str}}
\end{array}\right]\left[\begin{array}{l}
x_{\mathrm{RB}} \\
x_{\mathrm{Str}}
\end{array}\right]+D u .
\end{aligned}
$$

The Kalman filter design is carried out on the basis of the following rigid body model.

$$
\begin{aligned}
\dot{x}_{\mathrm{RB}} & =A_{\mathrm{RB}} x_{\mathrm{RB}}+B_{\mathrm{RB}} u+w_{1}, \\
y & =C_{\mathrm{RB}} x_{\mathrm{RB}}+D u+w_{2},
\end{aligned}
$$


$w_{1}$ and $w_{2}$ are the process noise and the measurement noise respectively, representing the actual noise of the system, as well as the portion of the output that corresponds to the structural modes. The covariance matrices $Q, R$ and $N$ are defined by:

$$
E\left\{\left[\begin{array}{l}
w_{1} \\
w_{2}
\end{array}\right]\left[\begin{array}{ll}
w_{1}^{\prime} & w_{2}^{\prime}
\end{array}\right]\right\}=\left[\begin{array}{cc}
Q & N \\
N^{\prime} & R
\end{array}\right] .
$$

To design the Kalman gain, $L$, the covariance matrices $Q, R$ and $N$ must be defined. Since the noise signals $w_{1}$ and $w_{2}$ include the portion of the output that correspond to the structural modes, the matrices $Q, R$ and $N$ are used as design parameters instead of being related to the actual noise power. There is a trade-off between robustness and performance that is controlled by the relative size of $Q$ and $R$. If $Q$ is much larger than $R$, the design relies more on the measured signal and therefore the structural modes are lightly filtered. If $Q$ is smaller than $R$, the rigid body model is trusted more than the measure signal and therefore the structural modes are heavily filtered. In the latter case, however, the design is less robust against rigid body model mismatch.

The Kalman filter is built based on the following rigid body portion:

$$
\begin{aligned}
& \dot{\hat{x}}_{\mathrm{RB}}=A_{\mathrm{RB}} \hat{x}_{\mathrm{RB}}+B_{\mathrm{RB}} u+L\left(y-\hat{y}_{\mathrm{RB}}\right), \\
& \hat{y}_{\mathrm{RB}}=C_{\mathrm{RB}} \hat{x}_{\mathrm{RB}}+D u .
\end{aligned}
$$

\subsection{Simulation results}

The block diagram for Kalman filter design used to decouple rigid body and structural modes is shown in figure 11. The inputs are the rudder and the aileron, while the outputs are the roll rate, yaw rate and lateral acceleration.

Open loop transfer functions: Figure 12 shows a comparison between the transfer function from $u$ to the actual output $y$ and from $u$ to the Kalman filter estimate of the rigid body output, $\hat{y}_{\mathrm{RB}}$. It is clear from the plot that the rigid body estimate reproduces the rigid body portion of the output in the low frequency range, and at the same time contains very little energy at higher frequencies, where most of the structural modes lie.

Closed loop transfer functions: Simplified diagrams of the current configuration and the Kalman filter configuration are shown in figure 2. Figure 13 shows the closed-loop transfer

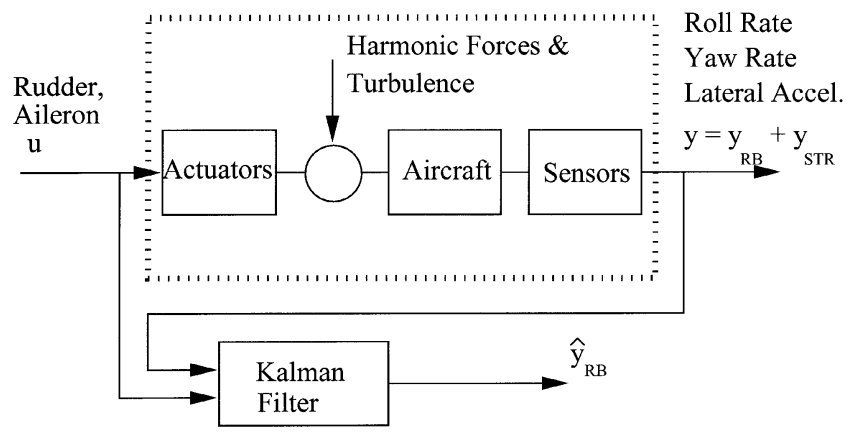

Figure 11. Open loop Kalman filter configuration for decoupling rigid body and structural modes. 
Open Loop Aileron to Roll Rate transfer function

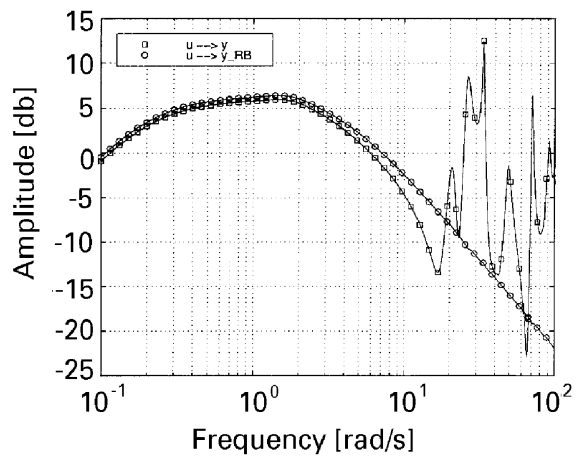

Open Loap Aileron to Lateral Accel transfer function

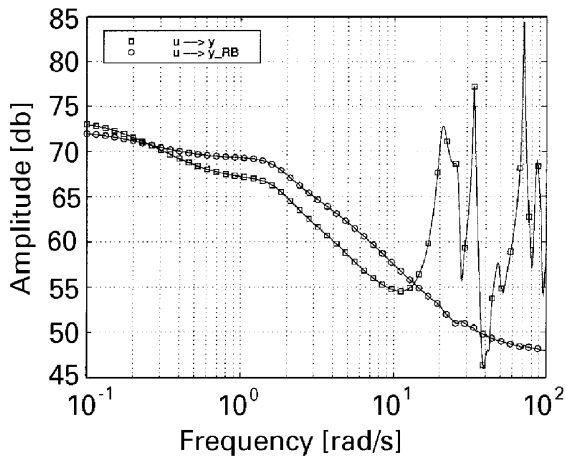

Open Loop Rudder to Yaw Rate transfer function

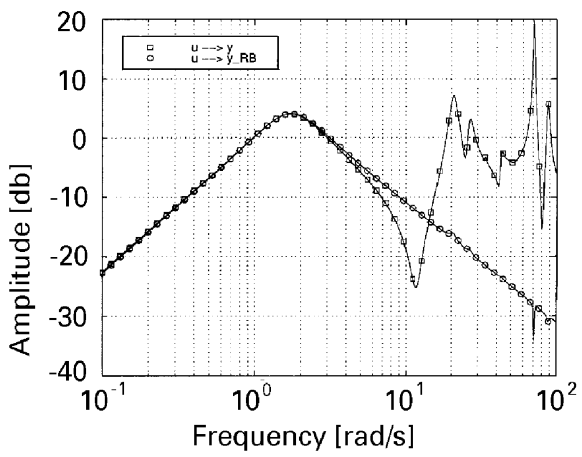

Open Loop Aileron to Yaw Rate transfer function

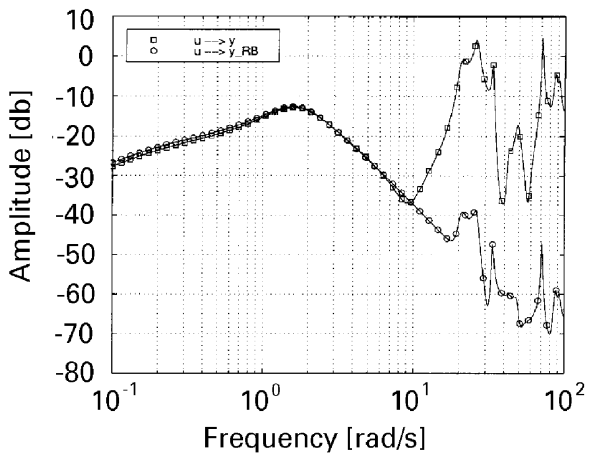

Open Loop Rudder to Roll Rate transfer function

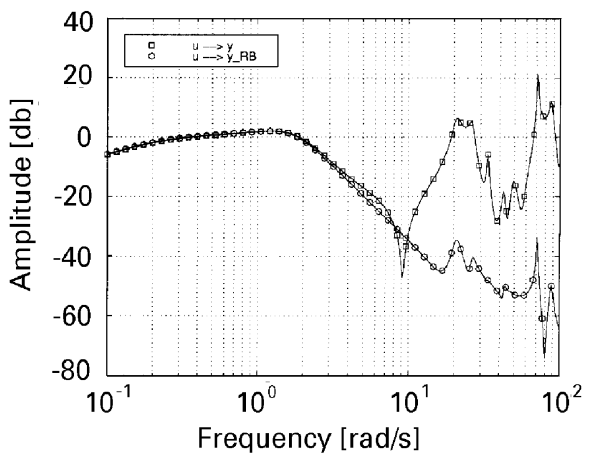

Open Loop Rudder to Lateral Accel transfer function

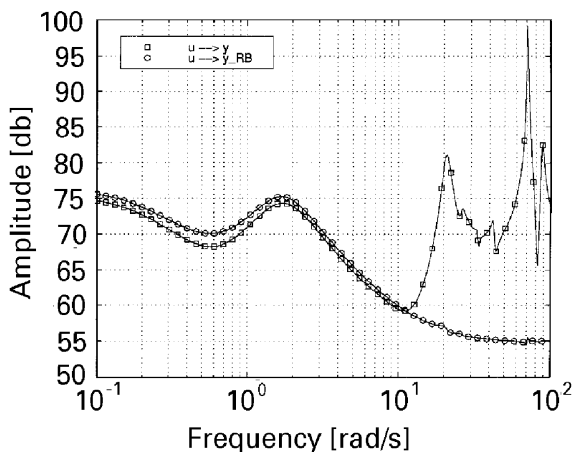

Figure 12. Open-loop transfer function amplitude from $u$ to the actual output, $y$, and from $u$ to the Kalman filter estimate of the rigid body portion, $\hat{y}_{\mathrm{RB}}$.

function amplitude from $v$ to $y$ for the two inputs - lateral stick and pedal - and the three outputs - roll rate, yaw rate and lateral acceleration sensors. In the first curve (solid line), the feedback path does not contain notch filters. This closed-loop system is unstable as several structural poles move to the right half plane. The second curve (square symbols) corresponds to the current configuration; that is, three notch filters are tuned at the critical structural mode frequencies. These filters were designed to suppress structural modes above $10 \mathrm{rad} / \mathrm{s}(1.5 \mathrm{~Hz})$, but they also affect the rigid body dynamics below $10 \mathrm{rad} / \mathrm{s}$. The most 


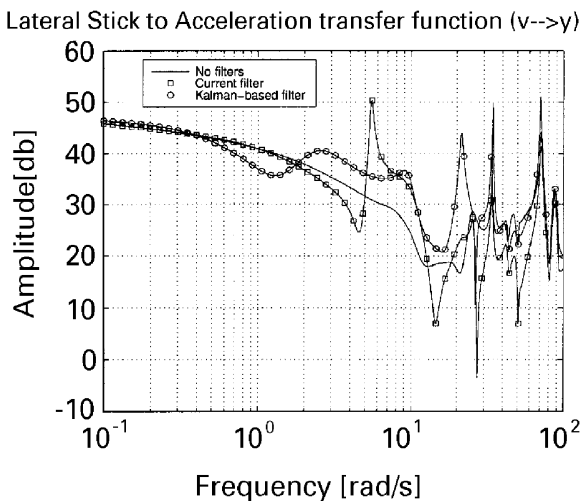

Lateral Stick to Yaw Rate transfer function ( $v-->y)$

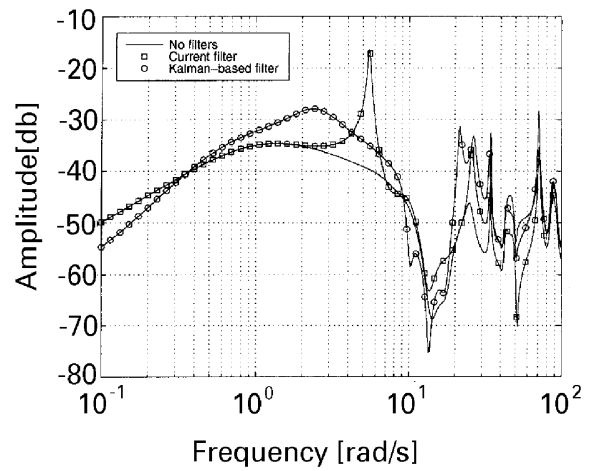

Pedal to Roll Rate transfer function ( $\mathrm{v-}-\mathrm{y})$

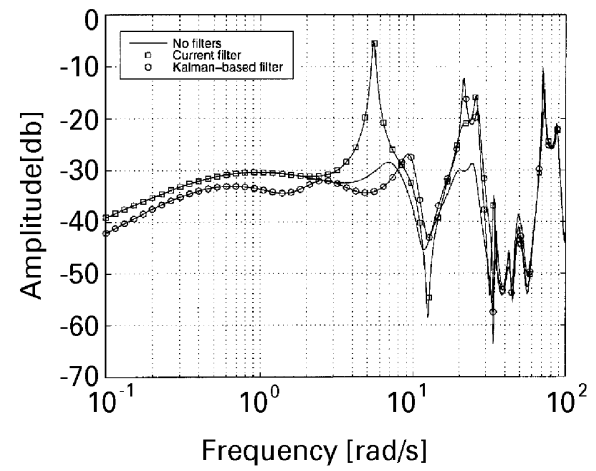

Lateral Stick to Roll Rate transfer function ( $v-->y)$

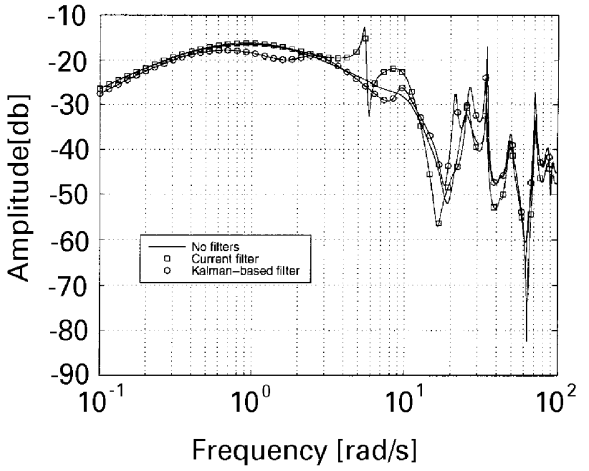

Pedal to Lateral Acceleration transfer function $(\mathrm{v}-\mathrm{-}>\mathrm{y})$

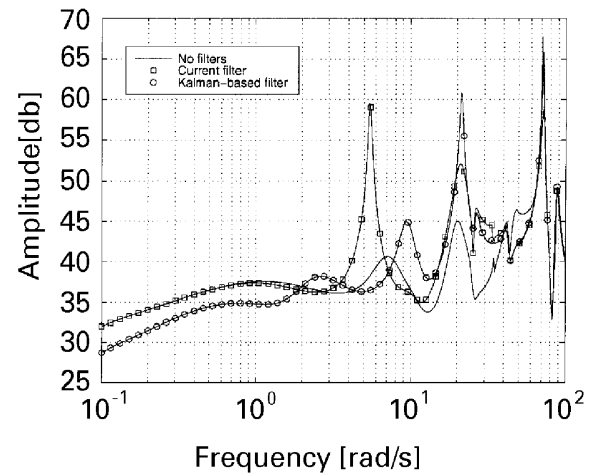

Pedal to Yaw Rate transfer function ( $v-->y)$

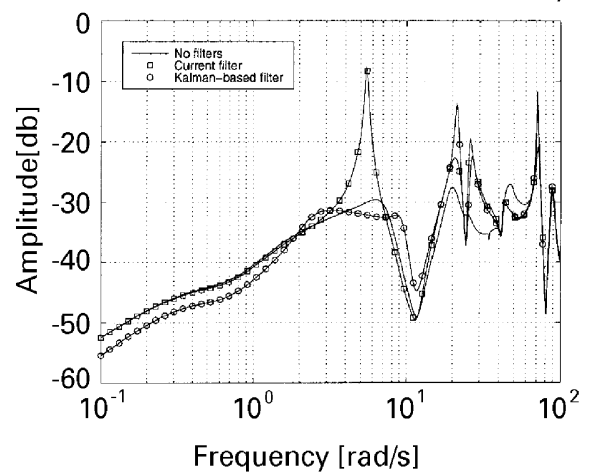

Figure 13. Closed-loop transfer function amplitude for the system without notch filters, for the current system with standard notch filters, and for the system with Kalman-based filter. The closed-loop system without notch filters is unstable while the other two are stable.

noticeable effect of the interaction between these notch filters and the rigid body dynamics is at a relatively high peak around $5.5 \mathrm{rad} / \mathrm{s}(0.9 \mathrm{~Hz})$ that appears after the loop is closed. This peak produces lightly damped time responses as shown in the next section. The third curve (open circles) shows the transfer function amplitude when the loop is closed using the 

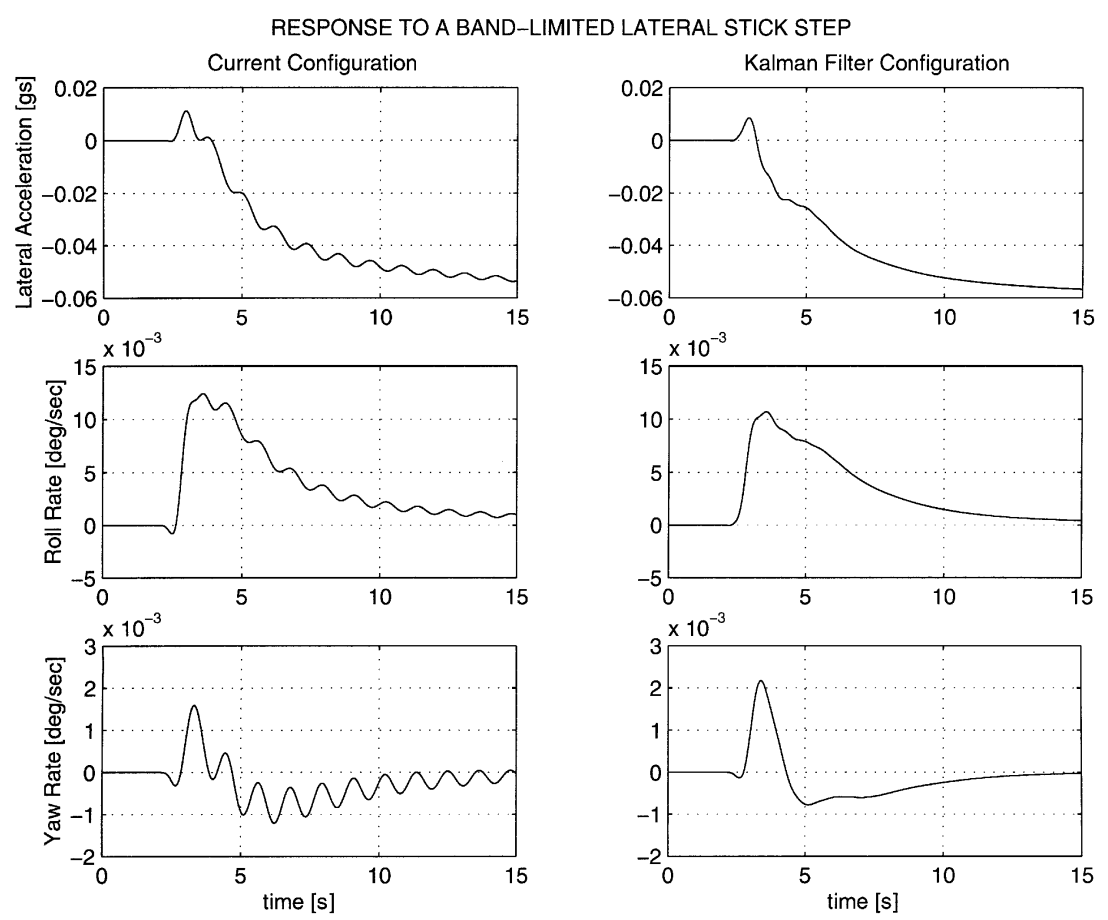

Figure 14. Time histories of the roll rate, yaw rate and lateral acceleration when the system is excited with a step lateral stick signal filtered by a $10 \mathrm{rad} / \mathrm{s}$ cut-off lowpass filter.

Kalman filter. In this case the structural modes change very little and stability is preserved. There is no prominent peak in the frequency range below $10 \mathrm{rad} / \mathrm{s}$, and therefore the transients vanish faster, as shown in the next section.

Time histories: Since the pilot is able to excite the system only up to a frequency of approximately $10 \mathrm{rad} / \mathrm{s}$, we compared the time histories of the current and the Kalman filter configurations by exciting the systems with a step signal which is filtered by a low pass filter with cut-off frequency of $10 \mathrm{rad} / \mathrm{s}$. Figures 14 and 15 show the roll rate, yaw rate and lateral acceleration responses when the loop is closed through both the baseline filters (current configuration) and the Kalman filter. It is apparent that the baseline-based closedloop response has strong oscillatory components due to the prominent peak around $5.5 \mathrm{rad} / \mathrm{s}$ exhibited by the closed-loop transfer function. In the case of the Kalman filter based configuration, the response is smoother because the closed-loop transfer function has no peaks in the range below $10 \mathrm{rad} / \mathrm{s}$.

\subsection{Pilot airframe coupling}

To analyse the improvement achieved by the use of Kalman filter based notching in ameliorating the Pilot Airframe Coupling, the phase delay of both the current configuration and Kalman filter based configuration was further investigated. Pilot Airframe Coupling may instabilize the system if the phase delay is large. 

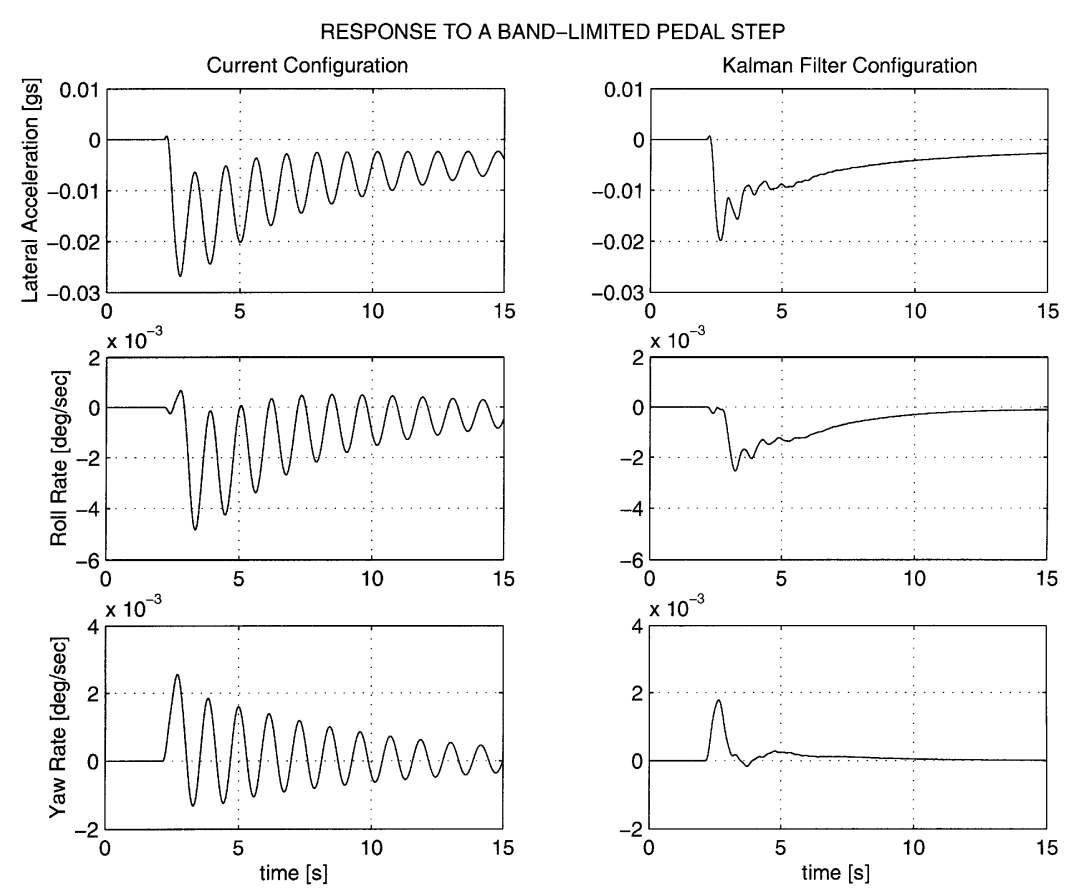

Figure 15. Time histories of the roll rate, yaw rate and lateral acceleration when the system is excited with a step pedal signal filtered by a $10 \mathrm{rad} / \mathrm{s}$ cut-off lowpass filter.

According to the US Aeronautical Design Standard for handling quality requirements, the phase delay, $\tau_{p}$, is defined as follows:

$$
\tau_{p}=\frac{\Delta \Phi 2 \omega_{180}}{57.3\left(2 \omega_{180}\right)},
$$

where $\omega_{180}$ is defined as the angular frequency at which the phase becomes $180^{\circ}$, while $\Delta \Phi 2 \omega_{180}$ is the phase increment (in absolute value) from $\omega_{180}$ to two times that frequency

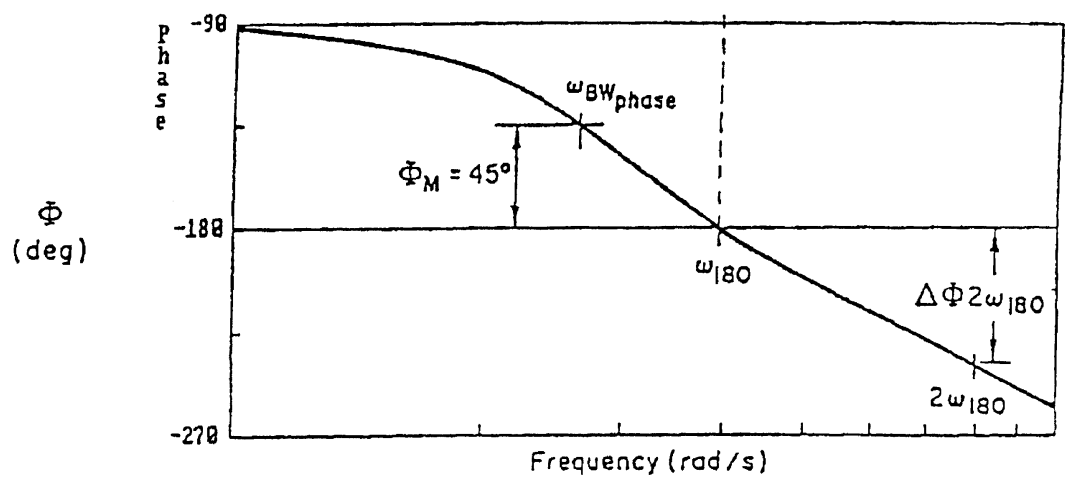

Figure 16. Parameters used in the definition of the phase delay, according to aeronautical standards. 


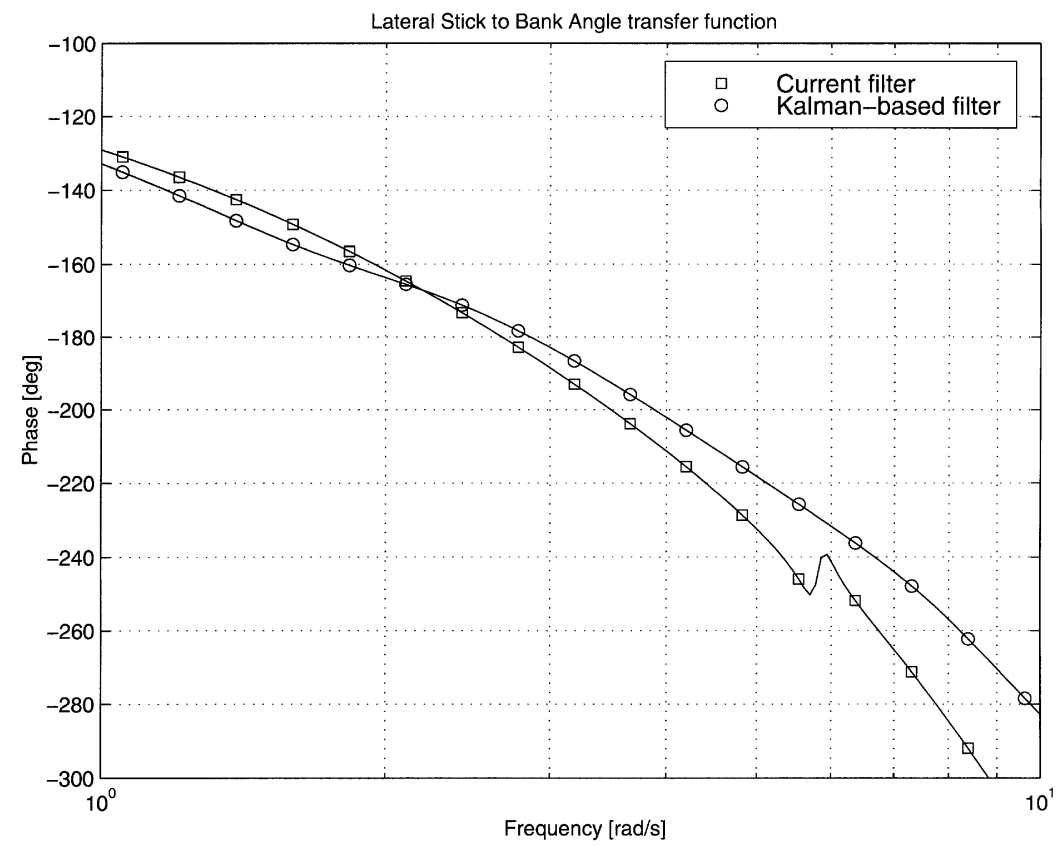

Figure 17. Phase of the lateral stick to bank angle transfer function for the cases of the current configuration and the Kalman filter based configuration.

(denoted as $2 \omega_{180}$ ) (see figure 16). Figure 17 shows the phase of the transfer function between the lateral stick input and the bank angle output for the cases of current configuration and Kalman filter based configuration. (The bank angle is defined as the integral of the roll rate.) The current configuration results in a phase delay of $\tau_{p}=0.19 \mathrm{~s}$, while the Kalman filter based configuration results in a phase delay of $\tau_{p}=0.14 \mathrm{~s}$.

To further analyse the effect of this phase delay in the pilot airframe coupling, we considered the block diagram in figure 18 - the block on the right hand side represents the aircraft, the flight control system (FCS), and the notch filters. The block on the left hand side represents pilot dynamics. The pilot is customarily represented as a gain and a delay. High gains are associated with high precision maneuvers, as the pilot must become more active to maintain small deviations from the desired trajectory. In these kinds of maneuvers, it is more likely that the pilot airframe coupling may produce undesired oscillations.

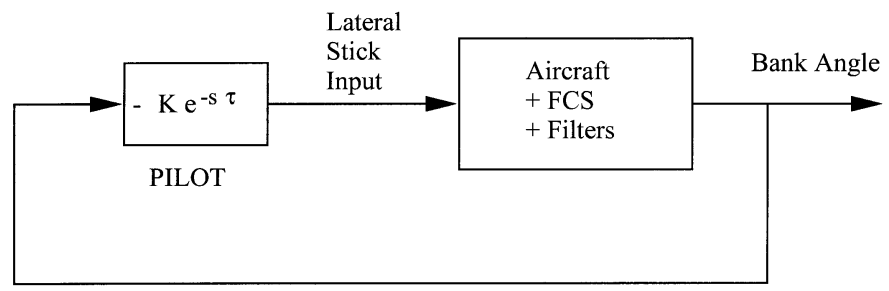

Figure 18. Block diagram to study the effect of different configurations in the pilot airframe coupling problem. 
Table 1. Maximum $K$ (pilot gain) after which instabilities occur.

\begin{tabular}{lcc}
\hline & \multicolumn{2}{c}{$K_{\max }$} \\
\cline { 2 - 3 }$\tau_{p}(\mathrm{~s})$ & Current configuration & Kalman filter \\
\hline 0 (no delay) & 23.4 & 34.2 \\
0.08 & 19.2 & 23.5 \\
\hline
\end{tabular}

To assess the handling qualities for each configuration, the maximum gain $\left(K_{\max }\right)$ that instabilizes the system in the block diagram of figure 18 is calculated for the typical values of no delay ( $\tau=0 \mathrm{~s}$ ) and a delay of $\tau=0.08 \mathrm{~s}$. The results are presented in table 1, which show that the Kalman filter based configuration allows for a larger pilot gain, therefore leading to improved handling qualities.

\section{Conclusions}

(1) DSRA has been shown to be a very effective method for off-line and on-line identification of aircraft structural modes. The method can be used with arbitrary input signals and only turbulence excited data. The use of DSRA instead of the Prony method can result in significant savings in structural flight testing because DSRA can simultaneously identify several modes.

(2) It is shown through simulations that the implementation of a self-adaptive notch filter for the AWC mode by means of the DSRA identification algorithm is feasible. The amplitude of the probe signal must be within certain values for pilot acceptability. If it is too low, the estimated frequency may drift as the notch filter is updated, producing undesirable effects. For the AWC mode, there is an excitation amplitude - around $50 \mathrm{lb}$ - that satisfies identification requirements. For this amplitude, the output signal grows to $0.25 \mathrm{~g}$ from $0.17 \mathrm{~g}$ approximately, which corresponds to the output that would result if the notch filter were fixed at the right frequency and no probe signals were injected. The bias in the estimation is small enough to maintain the stability of the closed loop, despite the relatively large variations in the AWC mode frequency.

(3) High fidelity simulations were carried out to further test the Kalman filter approach to reduce structural and rigid-body coupling. The closed-loop transfer function with the current set of notch filters and the closed-loop transfer function with the Kalman filter were compared. In the case of the current notch filter design in V-22, the transfer functions exhibited a prominent peak around $5.5 \mathrm{rad} / \mathrm{s}$ produced by the structural rigidbody coupling. This peak produces lightly damped time responses that degrades the flying quality. The Kalman filter approach instead produced smoother transfer functions in the frequency range up to $10 \mathrm{rad} / \mathrm{s}$.

(4) Further analysis showed that, as opposed to the current configuration, the Kalman filter approach does not increase the phase delay of the entire system. This, in turn allows for larger pilot gain required for high precision maneuvers, therefore leading to improved handling qualities.

This research was supported by US Navy through contract N62269-96-C-0030 from NAWC-Aircraft Division, Patuxent River, MD 20670, USA. 


\section{References}

Akaike H 1974 A new look at statistical model identification. IEEE Trans. Autom. Control AC-19: 716-723

Aoki M 1987 State space modeling of time series (Berlin: Springer Verlag)

Faure P 1976 Stochastic realization algorithms. In System Identification: Advances and Case Studies (eds) R K Mehra, D G Larniotis (New York: Academic Press)

Mehra R K 1991 Identification of state space models using a stochastic realization algorithm with applications to modal identification of flexible aerospace structures. IFAC Symp. on System Identification, Budapest, June

Mehra R K 1994a A new unified approach to state space model structure determination and maximum likelihood identification with aerospace applications. IFAC Symp. on System Identification, Copenhagen, July

Mehra R K 1994b Optimal input signals for parameter estimation in dynamical systems - survey and new results. IEEE Trans. Autom. Control AC-19: 753-768

Mehra R K, Arambel P O, Sampath A M, Prasanth R, Parham T C 1998 Self-adaptive notch filter for the V-22 flight controls using stochastic realization algorithm (SRA). Final Report, Contract N62269-96-C-0030, Scientific Systems Co.

Parham T C, Popelka D, Miller D G, Froebel A T 1991 V-22 pilot-in-the-loop aeroelastic stability analysis. American Helicopter Society 47th Annual Forum, May, Phoenix

van Overschee P, De Moor B 1993 Subspace algorithms for the stochastic identification problem. Automatica 29: 649-660

van Overschee P, De Moor B 1994 N4SID: Subspace algorithms for the identification of combined deterministic stochastic systems. Automatica 30: 75-93

Verhaegen M 1994 Identification of the deterministic part of MIMO state space models given in innovations form from input-output data. Automatica 30: 61-74

Viberg M, Ottersten B, Wahlberg B, Ljung L 1993 Performance of subspace based state-space system identification methods. Proc. 12th World Congress IFAC Sydney, 7: 369-372 